\title{
A DISTRIBUTIONAL REPRESENTATION OF STRIP ANALYTIC FUNCTIONS
}

\author{
DRAGIŜA MITROVIĆ \\ Department of Mathematics \\ Faculty of Technology \\ University of Zagreb \\ Zagreb, Yugoslavia \\ (Received January 16, 1981)
}

ABSTRACT. A strip analytic function converging in the $D^{\prime}$ topology to certain boundary values (from the interior of the strip) is represented as the difference of two generalized Cauchy integrals.

KEY WORDS AND PHRASES. Analytic function, distribution in D', distribution in $G^{\prime}$, convergence of distributions, Cauchy representation of a distribution I generalized Cauchy integral), Plemelj distributional formulas.

1980 MATHEMATICS SUBJECT CLASSIFICATION CODES. 46F20, 46F10, $30 E 20$.

1. INTRODUCTION.

In the theory of distributional behavior of analytic functions, two following topics are central: (1) the representation of distributions in terms of boundary values of analytic functions; (2) the representation of analytic functions in terms of distributions.

The present paper, influenced by [1, Theorem 97, p. 130] via [2, Theorem 3.6, p. 68], continues the note [3] and contributes to the second topic. In the cited theorem of Beltrami and Wohlers, there is established a decomposition of strip analytic functions into the difference of two Cauchy distributional representations concerning the $S^{\prime}$ topology. Here, a version of this boundary value theorem is proved involving the $D^{\prime}$ topology.

2. NOTATION AND PRELIMINARIES.

Throughout this paper the following symbols will be used: 
$t$ : the real coordinate of a point of $I R$;

$z, \zeta$ : the complex coordinates of points of $\phi, z=x+i y$;

$\Delta^{+}, \Delta^{-}:$the open upper half-plane $\{z \in \mathbb{Q}: \operatorname{Im}(z)>0\}$ and the open lower half-plane $\{z \in \phi: \operatorname{Im}(z)<0\}$ respectively;

$C^{\infty}=C^{\infty}(\mathbb{R})$ : the vector space of all infinitely differentiable complex valued functions defined on $\mid R$;

$D=D(\mathbb{R}):$ the vector space of all $C^{\infty}$-function with a compact support;

$D^{\prime}=D^{\prime}(\mathbb{R})$ : the space of all continuous linear functionals (Schwartz distributions) on $D$.

For the completeness we recall a few basic definitions and facts on the spaces $\epsilon_{\alpha}=\epsilon_{\alpha}(\mid R)$ and $\epsilon_{\alpha}^{\prime}=\epsilon_{\alpha}^{\prime}(\mathbb{R})$.

Let $\alpha$ be a real number. We say that a function $\phi \in G_{\alpha}$ if $\phi \in C^{\infty}$ and for each non-negative integer $p$ there exists a constant $M_{p}$ such that $\left|D^{p} \phi(t)\right| \leq M_{p}(1+|t|)^{\alpha}$ for all $t \in \mathbb{R}$. A sequence $\left(\phi_{n}\right)=\left(\phi_{n}\right)_{n} \in \mathbb{N}$ is said to converge to zero in $\epsilon_{\alpha}$ if the following are satisfied: (1) each $\phi_{\mathrm{n}} \in \mathfrak{G}_{\alpha}$; (2) for each $\mathrm{p}$ the sequence $\left(D^{\mathrm{P}} \phi_{n}\right)$ converges uniformly to zero on every compact subset of $\mathbb{R}$; (3) for each $p$ there exists a constant $M_{p}$, independent of $n$, such that $\left|D^{p} \phi_{n}(t)\right| \leq M_{p}(1+|t|)^{\alpha}$ for all $t \in \mid R$. The space $D$ is dense in $G_{\alpha}$ (that is, for each $\phi \in \epsilon_{\alpha}$ there exists a sequence $\left(\phi_{n}\right)$ in $D$ which converges to $\dot{\psi}$ in $\left.\epsilon_{\alpha}\right)$. A linear functional T on $G_{\text {into }} \mathbb{T}$ is continuous if $\lim _{\mathrm{n} \rightarrow \infty}\left\langle\mathrm{T}, \phi_{\mathrm{n}}\right\rangle=\left\langle\mathrm{T}, \lim _{\mathrm{n} \rightarrow \infty} \phi_{\mathrm{n}}\right\rangle=\langle\mathrm{T}, \phi\rangle$ for any sequence $\left(\phi_{n}\right)$ that converges to $\phi$ in $\epsilon_{\alpha}$. The space $c_{\alpha}^{\prime}$ is the space of all continuous linear functionals (distributions) on $c_{\alpha}$. Finally, note the proper inclusions $D \subset c_{\alpha}$ and $\epsilon_{\alpha}^{\prime} \subset D^{\prime}$.

In the following we shall use the same expression to denote a regular distribution and a function that generates it (when no confusion is possible).

3. AUXILIARY RESULTS.

In order to establish the main result, we shall need the following three simple lemmas.

LENAA 3.1: If $h^{+}(z)$ is a function analytic in $\Delta^{+}$with $h^{+}(z)=0\left(\left|\frac{1}{z}\right|\right.$ ) as $|z| \rightarrow \infty$ in $\Delta^{+}$, and if $h^{+}(x+i \varepsilon)$ converges to $h_{x}^{+}$in the $\mathcal{D}^{\prime}$ topology as $\varepsilon \rightarrow+0$, 
that is,

$$
\left\langle\mathrm{h}_{\mathrm{x}}^{+}, \phi\right\rangle=\lim _{\varepsilon \rightarrow+0}\left\langle\mathrm{~h}^{+}(\mathrm{x}+\mathrm{i \varepsilon}), \phi\right\rangle=\lim _{\varepsilon \rightarrow+0} \int_{-\infty}^{\infty} \mathrm{h}^{+}(\mathrm{x}+\mathrm{i \varepsilon}) \phi(\mathrm{x}) \mathrm{dx}
$$

for each $\phi \in D$, then $h_{x}^{+} \in \epsilon_{\alpha}^{\prime}$ for all $\alpha<0$.

PROOF. For each $\varepsilon>0$ the function $x \mapsto h^{+}(x+i \varepsilon)$ is continuous on $\mathbb{R}$.

Therefore for each $\varepsilon>0$ the linear functional on $\mathcal{D}$ into $\mathbb{C}$ defined by the integral

$$
\left\langle\mathrm{h}^{+}(\mathrm{x}+\mathrm{i \varepsilon}), \phi\right\rangle=\int_{-\infty}^{\infty} \mathrm{h}^{+}(\mathrm{x}+\mathrm{i} \varepsilon) \phi(\mathrm{x}) \mathrm{dx}
$$

is a regular distribution in $V^{\prime}$. By the hypothesis on the behavior of $\mathrm{h}^{+}(z)$ there exist the constants $R>0$ and $A>0$ such that for each $\varepsilon>0$ and all $|x|>R$ the inequality

$$
\left|h^{+}(x+i \varepsilon)\right| \leq \frac{A}{\sqrt{x^{2}+\varepsilon^{2}}}<\frac{A}{|x|}
$$

holds. Then for all $\phi \in D$ with a support contained in the set $E=\{x \in \mathbb{R}$ : $|x| \geq r>R\}$ it follows

$$
\left|\left\langle\mathrm{h}_{\mathrm{x}}^{+}, \phi\right\rangle\right|=\lim _{\varepsilon \rightarrow+0}\left|\int_{-\infty}^{\infty} \mathrm{h}^{+}(\mathrm{x}+\mathrm{i} \varepsilon) \phi(\mathrm{x}) \mathrm{dx}\right| \leq \mathrm{A} \int_{-\infty}^{\infty}|\mathrm{x}|^{-1}|\phi(\mathrm{x})| \mathrm{dx} .
$$

Thus the distribution $\mathrm{h}_{\mathrm{x}}^{+}$has the asymptotic bound $|\mathrm{x}|^{-1}$. Hence, by Theorem [4, p. 54] it can be extended from $D^{\prime}$ to $\mathbb{G}_{\alpha}^{\prime}$ for all $\alpha<0$. In other words, $\mathrm{h}_{\mathrm{x}}^{+} \in \dot{s}_{\alpha}^{\prime}(\alpha<0)$.

Also, since

$$
\left|\left\langle h^{+}(x+i \varepsilon), \phi\right\rangle\right| \leq A \int_{-\infty}^{\infty}|x|^{-1}|\phi(x)| d x
$$

for each $\varepsilon>0$ and all $\phi$ with Supp $\phi \subset E$, we conclude that $h^{+}(x+i \varepsilon)$ is a regular distribution in $\varsigma_{\alpha}^{\prime}(\alpha<0)$.

REMARK 3.1. Perhaps it may be of interest to prove the above result directly. Consider a linear functional on $\epsilon_{\alpha}(\alpha<0)$ defined by means of

$$
\left\langle h^{+}(x+i \varepsilon), \phi\right\rangle=\int_{-\infty}^{\infty} h^{+}(x+i \varepsilon) \phi(x) d x, \phi \in \xi_{\alpha} .
$$

For each $\varepsilon>0$ the integral (3.1) exists because the integrand is equal to 
$\rho\left(|x|^{-1+\alpha}\right)$. Let $\left(\phi_{n}\right)$ be any sequence which converges to zero in $\mho_{\alpha}$ as $n \rightarrow \infty$. We must show that

$$
\lim _{n \rightarrow \infty}\left\langle h^{+}(x+i \varepsilon), \phi_{n}\right\rangle=0
$$

Let $\mathrm{r}$ denote a positive real number. Then we can write

$$
\begin{gathered}
\left|\int_{-\infty}^{\infty} h^{+}(x+i \varepsilon) \phi_{n}(x) d x\right| \leq\left|\int_{|x| \leq r} h^{+}(x+i \varepsilon) \phi_{n}(x) d x\right|+ \\
\int_{|x|>r}\left|h^{+}(x+i \varepsilon) \phi_{n}(x)\right| d x .
\end{gathered}
$$

Letting $\delta$ be an arbitrarily small positive real number, we may choose the number $r$ so large $(r>R)$ that

$$
\int_{|x|>r}\left|h^{+}(x+i \varepsilon) \phi_{n}(x)\right| d x \leq A M_{0} \int_{|x|>r}|x|^{-1+\alpha} d x<\delta
$$

for all $\mathrm{n}$. The closed interval $[-\mathrm{r}, \mathrm{r}]$ being now fixed, it follows from the convergence of $\left(\phi_{n}\right)$ to zero in $G_{\alpha}$ and the Lebesgue dominated convergence theorem that

$$
\lim _{n \rightarrow \infty} \int_{|x| \leq r} h^{+}(x+i \varepsilon) \phi_{n}(x) d x=0
$$

The bound (3.3) and the limit (3.4) together show that the estimate (3.2) can be made arbitrarily small for larg e enough $\mathrm{n}$. Consequently, the linear functional (3.1) is a regular distribution in $\sigma_{\alpha}^{\prime}(\alpha<0)$.

The previous results suggest the following lemma.

LEMMA 3.2. If the function $\mathrm{h}^{+}(\mathrm{z})$ satisfies the conditions of Lemma 3.1 , then $\mathrm{h}^{+}(\mathrm{x}+\mathrm{i \varepsilon})$ converges to $\mathrm{h}_{\mathrm{x}}^{+}$in the $\sigma_{\alpha}^{\prime}(\alpha<0)$ topology as $\varepsilon \rightarrow+0$, that is,

$$
\left\langle\mathrm{h}_{\mathrm{x}}^{+}, \phi\right\rangle=\lim _{\varepsilon \rightarrow+0}\left\langle\mathrm{~h}^{+}(\mathrm{x}+\mathrm{i} \varepsilon), \phi\right\rangle=\lim _{\varepsilon \rightarrow+0} \int_{-\infty}^{\infty} \mathrm{h}^{+}(\mathrm{x}+\mathrm{i} \varepsilon) \phi(\mathrm{x}) \mathrm{dx}
$$

for each $\phi \in \mathcal{C}_{\alpha}(\alpha<0)$.

PROOF. Let $\alpha$ be a negative real number and let $r$ be as in the proof of Lemma

3.1. To consider the limit we write

$$
\int_{-\infty}^{\infty} h^{+}(x+i \varepsilon) \phi(x) d x=\int_{|x| \leq r} h^{+}(x+i \varepsilon) \phi(x) d x+\int_{|x|>r} h^{+}(x+i \varepsilon) \phi(x) d x,
$$


where $\phi \in \mathbb{G}_{\alpha}$. As each function $\phi$ is a $C^{\infty}$-function, for any given compact of $\mathbb{R}$ there exists a function in $D$ that is identical to $\phi$ over this compact [6, p. 4]. So, by the hypothesis the first limit exists. Since $h^{+}(z)$ is analytic and bounded in the domain $\left\{z \in \Delta^{+}:|\operatorname{Re}(z)| \geq \mathrm{r}>\mathrm{R}\right\}$ it follows that $\mathrm{h}^{+}(\mathrm{x}+\mathrm{i \varepsilon}) \rightarrow \mathrm{h}^{+}(\mathrm{x})$ for almost all $|x| \geq r$ as $\varepsilon \rightarrow+0$. At the same time $\left|h^{+}(x+i \varepsilon)\right|<\frac{A}{|x|}$ for all $\varepsilon>0$. Therefore, using the Lebesgue dominated convergence theorem,

$$
\lim _{\varepsilon \rightarrow+0} \int_{|x|>r} h^{+}(x+i \varepsilon) \phi(x) d x=\int_{|x|>r} h^{+}(x) \phi(x) d x \in \mathbb{C} .
$$

Consequently, there exists a distribution $H \in \mathbb{s}_{\alpha}^{\prime}(\alpha<0)$ such that $\left\langle H_{x}, \phi\right\rangle=\lim _{\varepsilon \rightarrow+0}\left\langle h^{+}(x+i \varepsilon), \phi\right\rangle$ for each $\phi \epsilon G_{\alpha}$. This implies $h_{x}^{+}=H_{x}$ over D. But $D$ is dense in $G_{\alpha}$. Hence, $h_{x}^{+}=H_{x}$ over $G_{\alpha}$.

Obviously, the obtained results can be transposed bodily for a function $\mathrm{h}^{-}(\mathrm{z})$ analytic in $\Delta^{-}$with $\mathrm{h}^{-}(z)=0\left(\frac{1}{|z|}\right)$ as $|z| \rightarrow \infty$ and generating a regular distribution in $D^{\prime}$ by the integral

$$
\left\langle h^{-}(x-i \varepsilon), \phi\right\rangle=\int_{-\infty}^{\infty} h^{-}(x-i \varepsilon) \phi(x) d x .
$$

LEMMA 3.3. If the function $h^{t}(z)$ satisfies the condition of Lemma 3.1 , then

$$
\begin{array}{rlrl}
\frac{1}{2 \pi i}\left\langle h_{t}^{+}, \frac{1}{t-z}\right\rangle & =h^{+}(z) & \text { for } z \in \Delta^{+}, \\
& =0 \quad \text { for } z \in \Delta^{-} .
\end{array}
$$

PROOF. From Lemma 3.1 we know, in particular, that the distribution $h_{t}^{+}$acts on the space $\mathbb{G}_{\alpha}$ with $\alpha=-1$. Since the function $t \leftrightarrow \frac{1}{t-z}$ belongs to this space $(\operatorname{Im}(z) \neq 0)$, the Cauchy representation of $h_{t}^{+}$is well defined. To prove the lemma, we shall first evaluate the limit of the integral

$$
\frac{1}{2 \pi i}\left\langle h^{+}(t+i \varepsilon), \frac{1}{t-z}\right\rangle
$$

as $\varepsilon \rightarrow+0$ (observe that this integral exists for each $\varepsilon>0$ ). Let $z$ be any point in $\Delta^{+}$. By the Cauchy integral formular applied to the function

$$
\zeta \mapsto \frac{h^{+}(\zeta+i \varepsilon)}{\zeta-z}
$$

along the closed path consisting of a sufficiently large semicircle in $\Delta^{+}$of 
radius $r$ and the segment $[-r, r]$, we get

$$
\frac{1}{2 \pi i} \int_{-\infty}^{\infty} \frac{h^{+}(t+i \varepsilon)}{t-z} d t=h^{+}(z+i \varepsilon) \text { for } z \in \Delta^{+} \text {. }
$$

For $z \in \Delta^{-}$this integral vanishes. Thus, letting $\varepsilon \rightarrow+0$, we have

$$
\begin{aligned}
& \frac{1}{2 \pi i} \lim _{\varepsilon \rightarrow+0}\left\langle h^{+}(t+i \varepsilon), \frac{1}{t-z}\right\rangle=h^{+}(z) \text { for } z \in \Delta^{+} \text {, } \\
& =0 \text { for } z \in \Delta^{-} \text {. }
\end{aligned}
$$

Now by Lemna 3.2 the representation (3.5) follows.

For a function $\mathrm{h}^{-}(\mathrm{z})$ analytic in $\Delta^{-}$and satisfying here the conditions similar to ones of $\mathrm{h}^{+}(\mathrm{z})$, we infer by the same procedure that

$$
\begin{aligned}
& -\frac{1}{2 \pi i}\left\langle h_{t}^{-}, \frac{1}{t-z}\right\rangle=h^{-}(z) \text { for } \quad z \in \Delta^{-}, \\
& =0 \text { for } z \in \Delta^{+} \text {. }
\end{aligned}
$$

\section{THE MAIN RESULT.}

We are now prepared to prove the main result of this paper.

THEOREM 4.1. Let $\mathrm{f}(\mathrm{z})$ be a function analytic in the strip $\Delta=\{\mathbf{z} \in \mathbb{C}$ :

$\left.\mathrm{y}_{1}<\operatorname{Im}(\mathrm{z})<\mathrm{y}_{2}\right\}$ with $\mathrm{f}(\mathrm{z})=0\left(\frac{1}{|z|^{1+\lambda}}\right)$ for some $\lambda>0$ as $|z| \rightarrow \infty$ in $\Delta$. Suppose that $f_{1}=\lim _{\varepsilon \rightarrow+0} f\left(x+i\left(y_{1}+\varepsilon\right)\right)$ and $f_{2}=\lim _{\varepsilon \rightarrow+0} f\left(x+i\left(y_{2}-\varepsilon\right)\right)$ in the $D^{\prime}$ topology. Then for $y_{1}<\operatorname{Im}(z)<y_{2}$

$$
f(z)=\frac{1}{2 \pi i}\left\langle f_{1}, \frac{1}{t+i y_{1}-z}\right\rangle-\frac{1}{2 \pi i}\left\langle f_{2}, \frac{1}{t+i y_{2}-z}\right\rangle \text {, }
$$

where the Cauchy representation of $f_{1}$ is analytic in the upper half-plane $\operatorname{Im}(z)>y_{1}$, and the Cauchy representation of $f_{2}$ is analytic in the lower half-plane $\operatorname{Im}(z)<y_{2}$.

PROOF. Let $y_{1}<a<b<y_{2}$. Since $f(z)$ tends uniformly to zero as $|z| \rightarrow \infty$ in $\Delta$, an application of Cauchy's integral formula [7, Lemma 1, p.293] leads to the decomposition $f(z)=f^{+}(z)+f^{-}(z)$, where

$$
\begin{aligned}
& f^{+}(z)=\frac{1}{2 \pi i} \int_{-\infty+i a}^{\infty+i a} \frac{f(\zeta)}{\zeta-z} d \zeta, \\
& f^{-}(z)=-\frac{1}{2 \pi i} \int_{-\infty+i b}^{\infty+i b} \frac{f(\zeta)}{\zeta-z} d \zeta .
\end{aligned}
$$


We recall that the function $\mathrm{f}^{+}(z)$ is analytic in the upper half-plane $\operatorname{Im}(z)>a$, and $\mathrm{f}^{-}(\mathrm{z})$ is analytic in the lower half-plane $\operatorname{Im}(z)<\mathrm{b}$. By virtue of the arbitrarily closeness of the points $a$ and $b$ to the points $y_{1}$ and $y_{2}$ respectively, the strip $\Delta$ is the common domain of analyticity for $f^{+}(z)$ and $f^{-}(z)$. In order to investigate the behavior of these functions at the point at infinity consider the equality

$$
\begin{gathered}
z f^{+}(z)=\frac{1}{2 \pi i} \int_{-\infty+i a}^{\infty+i a} \frac{z f(\zeta)}{\zeta-z} d \zeta \\
=\frac{1}{2 \pi i} \int_{-\infty+i a}^{\infty+i a} \frac{\zeta f(\zeta)}{\zeta-z} d \zeta-\frac{1}{2 \pi i} \int_{-\infty+i a}^{\infty+i a} f(\zeta) d \zeta .
\end{gathered}
$$

The integral of the Cauchy type in (4.2) vanishes as $|z| \rightarrow \infty$ in the upper halfplane $\operatorname{Im}(z)>y_{1}$, while other one converges since $f(\zeta)=0\left(\frac{1}{|\zeta|^{1+\lambda}}\right), \lambda>0$. From this we conclude that $f^{+}(z)=0\left(\frac{1}{|z|}\right)$ as $|z| \rightarrow \infty$. Also, from a similar integral representation for $z f^{-}(z)$ we infer $f^{-}(z)=0\left(\frac{1}{|z|}\right)$ as $|z| \rightarrow \infty$ in the lower half-plane $\operatorname{Im}(z)<y_{2}$.

Further, we must verify that the functions $f^{+}(z)$ and $f^{-}(z)$ really converge in the $D^{\prime}$ topology to certain boundary values on $\operatorname{Im}(z)=y_{1}$ and $\operatorname{Im}(z)=y_{2}$ respectively (from the interior of $\Delta$ ). Let $z=x+i(a+\varepsilon)$ be a point in the half-plane $\operatorname{Im}(z)>a$. Then in the distributional setting

$$
\begin{aligned}
f^{+}(x+i(a+\varepsilon))= & \frac{1}{2 \pi i}\left\langle f(t+i a), \frac{1}{t-(x+i \varepsilon)}\right\rangle \\
f^{+}\left(x+i\left(y_{1}+\varepsilon\right)\right)= & \quad \lim _{a \rightarrow y_{1}} f^{+}(x+i(a+\varepsilon)) \\
& \quad \lim \frac{1}{2 \pi i}\left\langle f(t+i a), \frac{1}{t-(x+i \varepsilon)}\right\rangle \\
& a y_{1}
\end{aligned}
$$

By Lemma 3.1 the analyticity of $f(z)=0\left(\frac{1}{|z|}\right)$ in $\Delta(|z| \rightarrow \infty)$ and the convergence of $\mathrm{f}(\mathrm{t}+\mathrm{ia})$ to $\mathrm{f}_{1}$ as $a \rightarrow \mathrm{y}_{1}$ together imply $\mathrm{f}_{1} \in \mathfrak{G}_{\alpha}^{\prime}(-1 \leq \alpha<0)$. On the other hand, according to Lemma 3.2 we have

$$
f^{+}\left(x+i\left(y_{1}+\varepsilon\right)\right)=\frac{1}{2 \pi i}\left\langle f_{1}, \frac{1}{t-(x+i \varepsilon)}\right\rangle \text {. }
$$


Now, in view of the distributional Plemelj formulas [5, Theorem 2] we get

$$
\mathrm{f}_{\mathrm{x}}^{+}=\lim _{\varepsilon \rightarrow+0} \mathrm{f}^{+}\left(\mathrm{x}+\mathrm{i}\left(\mathrm{y}_{1}+\varepsilon\right)\right)=\frac{1}{2} \mathrm{f}_{1}-\frac{1}{2 \pi i}\left\langle\mathrm{f}_{1} * \mathrm{vp} \frac{1}{\mathrm{x}}\right\rangle
$$

in the $D^{\prime}$ topology.

Let $z=x+i(b-\varepsilon)$ be a point in the half-plane $\operatorname{Im}(z)<y_{2}$. Starting from

$$
f^{-}(x+i(b-\varepsilon))=-\frac{1}{2 \pi i}\left\langle f(t+i b), \frac{1}{t-(x-i \varepsilon)}\right\rangle
$$

and proceeding along the same lines as before, we find

$$
\begin{gathered}
f^{-}\left(x+i\left(y_{2}-\varepsilon\right)\right)=-\frac{1}{2 \pi i}\left\langle f_{2}, \frac{1}{t-(x-i \varepsilon)}\right\rangle, \\
E_{x}^{-}=\underset{\varepsilon \rightarrow+0}{1 i m} E^{-}\left(x+i\left(y_{2}-\varepsilon\right)\right)=\frac{1}{2} f_{2}+\frac{1}{2 \pi i}\left\langle f_{2} * v p \frac{1}{x}\right\rangle
\end{gathered}
$$

in the $D^{\prime}$ topology.

So we have proved that the function $f^{+}(z)$ [resp. $f^{-}(z)$ ] is analytic in the half-plane $\operatorname{Im}(z)>y_{1}$ [resp. $\left.\operatorname{Im}(z)<y_{2}\right]$ with the order relation $0\left(\frac{1}{|z|}\right)$ as $|z| \rightarrow \infty$, and that it converges in the $D^{\prime}$ topology to $f_{x}^{+}$on $\operatorname{Im}(z)=y_{1}\left[\operatorname{resp}\right.$. $f_{x}^{-}$on $\left.\operatorname{Im}(z)=y_{2}\right]$. In view of Lemma $3 \cdot 3$, it follows that

$$
\begin{array}{rlrl}
\frac{1}{2 \pi i}\left\langle f_{t}^{+}, \frac{1}{t+i y_{1}-z}\right\rangle & =f^{+}(z) & \text { for } & \operatorname{Im}(z)>y_{1}, \\
& =0 \quad \text { for } \quad \operatorname{Im}(z)<y_{1} .
\end{array}
$$

Analogously,

$$
\begin{aligned}
-\frac{1}{2 \pi i}\left\langle f_{t}^{-}, \frac{1}{t+i y_{2}-z}\right\rangle & =f^{-}(z) \text { for } \operatorname{Im}(z)<y_{2}, \\
& =0 \text { for } \operatorname{Im}(z)>y_{2} .
\end{aligned}
$$

Now we shall compute the value of the integral

$$
\frac{1}{2 \pi i}\left\langle f^{+}\left(t+i y_{2}\right), \frac{1}{t+i y_{2}-z}\right\rangle
$$

for $\operatorname{Im}(z)<y_{2}$. For such $z$ the function

$$
\zeta \mapsto \frac{f^{+}(\zeta)}{\zeta-z}
$$

is analytic inside the closed path which consists of the segment $\left[-r+i y_{2}\right.$, $\left.r+\mathrm{iy}_{2}\right]$ and the semicircle $\mathrm{L}_{\mathrm{r}}$ of radius $\mathrm{r}$ lying in $\operatorname{Im}(z)>y_{2} \cdot \operatorname{According}$ to Cauchy integral theorem, we may write

$$
\frac{1}{2 \pi i} \int_{-r}^{r} \frac{f^{+}\left(t+i y_{2}\right)}{t+i y_{2}-z} d t+\frac{1}{2 \pi i} \int_{L_{r}} \frac{f^{+}(\zeta)}{\zeta-z} d \zeta=0 .
$$


The integral along $\mathrm{L}_{\mathrm{r}}$ tends to zero as $\mathrm{r} \rightarrow \infty$. Thus the integral (4.3) is equal to zero for $\operatorname{Im}(z)<y_{2}$. Also, as an immediate consequence of the derivation above,

$$
\frac{1}{2 \pi i}\left\langle f^{-}\left(t+i y_{1}\right), \frac{1}{t+i y_{1}-z}\right\rangle=0
$$

for $\operatorname{Im}(z)>y_{1}$. Combining the Cauchy representation of $f_{t}^{+}$and $f_{t}^{-}$with $(4.4)$ and (4.3) respectively, we have

$$
\begin{aligned}
& f^{+}(z)=\frac{1}{2 \pi i}\left\langle f_{t}^{+}+f^{-}\left(t+i y_{1}\right), \frac{1}{t+i y_{1}-z}\right\rangle \text { for } \operatorname{Im}(z)>y_{1}, \\
& f^{-}(z)=-\frac{1}{2 \pi i}\left\langle f_{t}^{-}+f^{+}\left(t+i y_{2}\right), \frac{1}{t+i y_{2}-z}\right\rangle \text { for } \operatorname{Im}(z)<y_{2} .
\end{aligned}
$$

From the decomposition $f(z)=f^{+}(z)+f^{-}(z)$ we see that $f_{1}=f_{t}^{+}+f^{-}\left(t+i y_{1}\right)$ is the boundary value of $f(z)$ on $\operatorname{Im}(z)=y_{1}$ in the $D^{\prime}$ topology and $f_{2}=f_{t}^{-}+$ $\mathrm{f}^{+}\left(\mathrm{t}+\mathrm{iy_{2 }}\right)$ is the boundary value of $\mathrm{f}(\mathrm{z})$ on $\operatorname{Im}(z)=\mathrm{y}_{2}$ in the same topology. Consequent1y,

$$
\begin{aligned}
& f^{+}(z)=\frac{1}{2 \pi i}\left\langle f_{1}, \frac{1}{t+i y_{1}-z}\right\rangle \text { for } \operatorname{Im}(z)>y_{1}, \\
& f^{-}(z)=-\frac{1}{2 \pi i}\left\langle f_{2}, \frac{1}{t+i y_{2}-z}\right\rangle \text { for } \operatorname{Im}(z)<y_{2} .
\end{aligned}
$$

Again returning to the decomposition of the function $f(z)$, the representation ( 4.1$)$ follows at once.

\section{REFERENCES}

1. TITCHMARSH, E.C. Introduction to the theory of Fourier integrals, 2nd., Oxford University Press (1948).

2. BELTRAMI, E.J. and WOHLERS, M.R. Distributions and the boundary values of analytic functions, Academic Press, New York (1966).

3. MITROVIĆ, D. A distributional representation of analytic function, Math. Balkanica 4 , (1974), pp. 437-440.

4. BREMERMANN, H.J. Distributions, complex variables, and Fourier transforms, Addison-Wesley, New York (1965).

5. MITROVIĆ, D. The Plemelj distributional formulas, Bull. Amer. Math. Soc. $\underline{77}$ (1971) pp. 562-563.

6. ZEMANIAN, A.H. Distribution theory and transform analysis, Mc Graw-Hill, New York (1965).

7. SVESHNIKOV, A. and TIKHONOV, A. The theory of functions of a complex variable, Mir Publisher, Moscow (1978). 


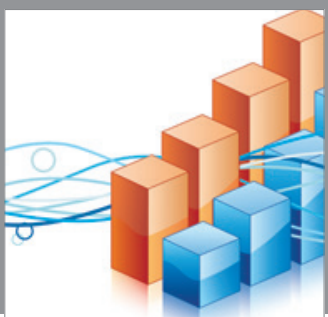

Advances in

Operations Research

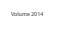

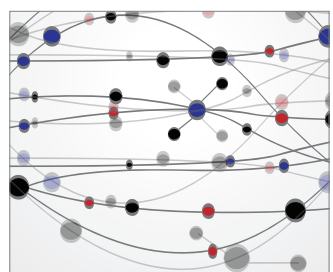

\section{The Scientific} World Journal
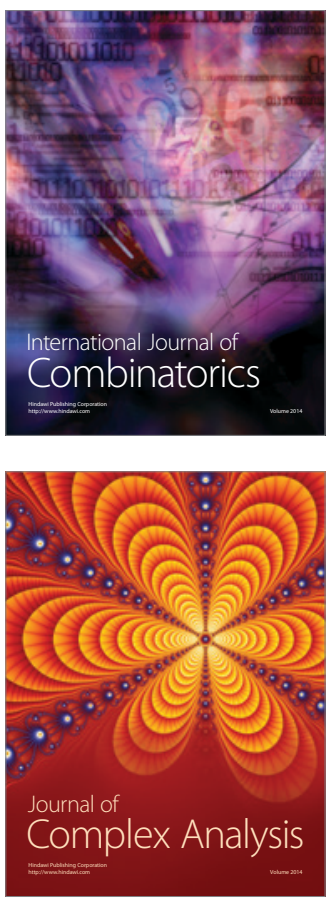

International Journal of

Mathematics and

Mathematical

Sciences
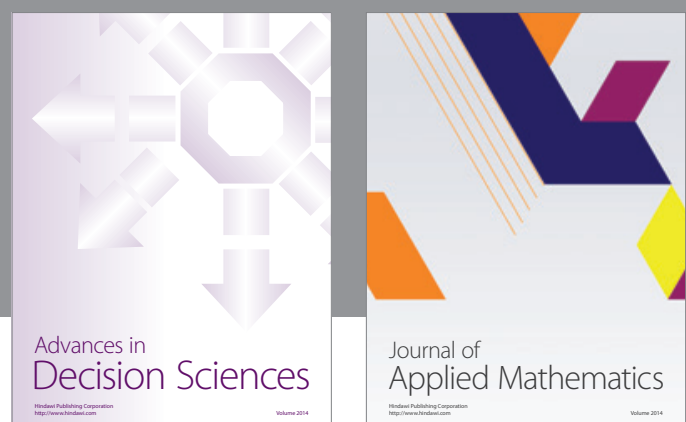

Journal of

Applied Mathematics
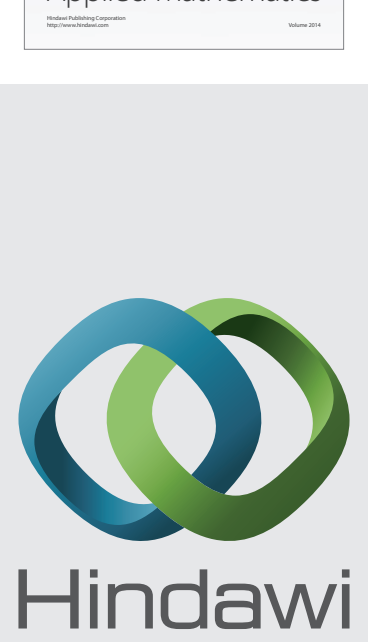

Submit your manuscripts at http://www.hindawi.com
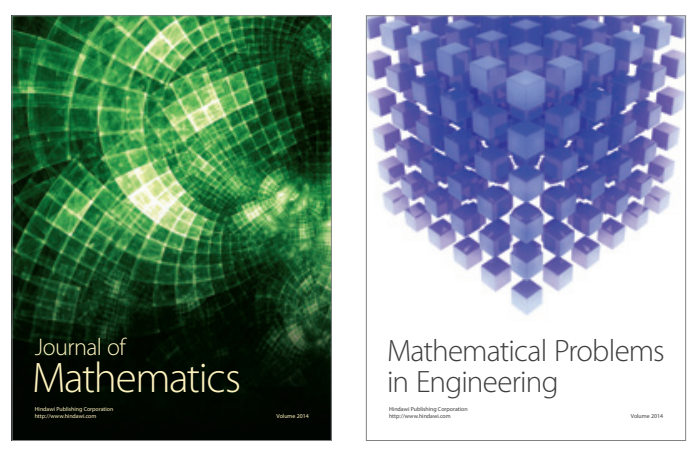

Mathematical Problems in Engineering
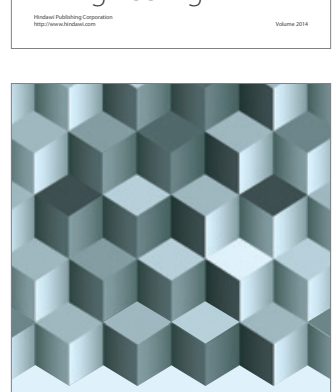

Journal of

Function Spaces
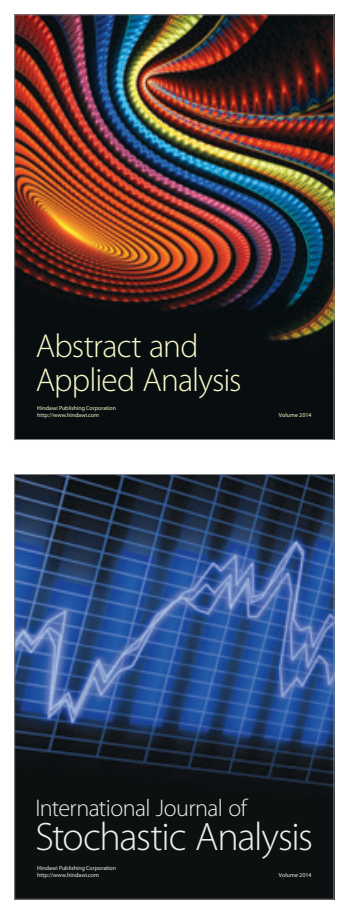

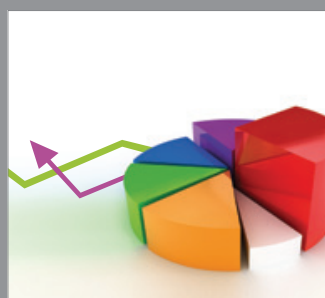

ournal of

Probability and Statistics

Promensencen
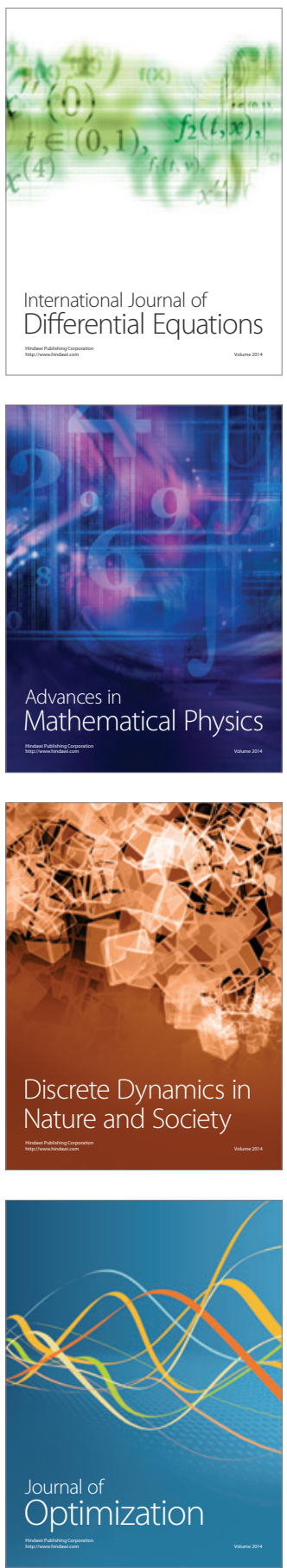